\title{
Role of Caspase -3 and Total Antioxidant Capacity (TAC) in Idiopathic Infertile Male Madha Mohammed Sheet Saleh $(\mathrm{PhD})^{1}$, Estabraq Abdulrasool Al -Wasiti $(\mathrm{PhD})^{2}$ and Adnan Nafil Muslim (Msc)
}

\begin{abstract}
Background: Sperm differentiation during spermatogenesis is highly regulated by different caspases and oxidant-antioxidant mechanisms.

Objective: To assess the levels of the caspase -3 and total antioxidant capacity (TAC) in seminal plasma and evaluate its role in the pathogenesis of idiopathic infertile male in comparison with healthy individuals.

Patients and Methods: Seminal fluids were collected from seventy males with idiopathic infertility and twenty male with proven fertility and were analyzed for the level of caspase -3 and TAC in seminal fluid and their correlation with seminal fluid quality parameters.

Results: The results revealed that men with idiopathic infertility have significantly higher level of caspase-3 activity. Seminal TAC levels were significantly lower in those with idiopathic infertility compared with fertile men. A negative correlation was noticed between sperm quality parameters and TAC levels in idiopathic infertile men.

Conclusion: Increased apoptotic index and decreased TAC activity are two important markers in idiopathic male infertility.

Key words: Caspase, idiopathic male infertility, Total antioxidant capacity (TAC).

Corresponding Author: adnannafil1981@gmail.com

Received: $14^{\text {th }}$ November 2017

Accepted: $28^{\text {th }}$ December 2017

https://doi.org/10.26505/DJM

${ }^{1,3}$ Middle Technical University- College of Health and Medical Technology- Baghdad- Iraq.

${ }^{2}$ College of Medicine- Al-Nahrain University- Baghdad-Iraq.

\section{Introduction}

Male infertility considered a central health issue that is regarded as globally problem and represent about $40 \%$ of the reproductive failure among married couples [1]. The etiology of male infertility is obviously a multifactorial one, could be pathological, physiological, nutritional, accidental or even immunological [2]. A high percentage of infertile men fail to impregnate their female counterpart because of the low quality of their seminal fluid parameters due to oligozoospermia, asthenozoospermia, teratozoospermia or azoospermia with

obscured or unknown reasons which is socalled idiopathic male infertility[3].

Principally, the imponderables between oxidants reactive oxygen species (ROS) and their scavenging or neutralization by the antioxidant system is considered as one of the risk factors in male infertility. However, it is believed that seminal oxidative stress could be one of the main factors in the pathogenesis of idiopathic infertility [4,5]. It is believed that controlled levels (normal or physiological levels) of these ROS are important for sperm normal physiology and
\end{abstract}


fertilization whereas, uncon 7 trolled or high production of ROS (abnormal or at pathological levels) may participate in sperm dysfunctions [6].In male who fertile, the production of oxidants and antioxidant remain in equilibrium status[7]. Naturally, antioxidants found in seminal fluid include vitamins, superoxide dismutase, glutathionas and thioredoxin [8].

High contents of polyunsaturated fatty acids (PUFAS) are accumulated in the spermatozoa plasma membranes, which would predis $\neg$ pose to oxidative injury. In addition, ROS could also induce base alterations, DNA cross-links, DNA strand breaks and chromosomal rearrangements [9].

For that reason, several investigantions have attempted to study the effects of antioxidant supplements on male infertility. Although the effects of different doses and types of oral antioxidants in attempts to improve seminal fluid parameters have not been established, it is believed that antioxidant therapy has beneficial ef $\neg$ fects on male infertility [10,11].Moreover, the results of antioxidant therapy alone or in combination are controversial. For example, some studies have observed that vita $\neg$ min $\mathrm{C}$, vitamin $\mathrm{E}$,folic acid, zinc and selenium alone or in combination could improve seminal fluid parameters, espe $\neg$ cially sperm motility and sperm DNA integrity[12-14].

Programmed cell death or apoptosis is a fundamental, very tightly and complex network of biochemical and molecular events with fine regulatory mechanisms that control the death event in cells. Various pathological situations such as cancer, autoimmune diseases and others can cause dysregulation of these processes that leads to cell death $[15,16]$. This suicidal pathway carried out or mediated by many proteolytic enzymes called caspases enzymes that exists in all cells as inactive form. Caspases (cysteinyl aspartate-specific proteinases) are a family of proteases that containing cysteine at their active sites. These proteolytic enzymes has a crucial role in induction cell death by cleaving specific proteins in the cytoplasm and nucleus [17].Principally, there are two major pathways which were described in the apoptosis induction mechanism including extrinsic or death receptor pathway (FasL, Fas mechanism) and intrinsic or mitochondrial pathway that triggered in response to a wide range of death stimuli that are generated from within the cell, such as oncogene activation and DNA damage [18]. The extrinsic pathway or death receptor pathway is normally defined by caspase- 8 activation (initiator caspase) which is a cysteinyl-aspartate protease that recruited by the adapter molecules Fas-Associated protein with Death Domain (FADD). These adapter molecules are associated with the domain of death receptors such as FAS, tumor necrosis factor-receptor 1 (TNF-R1) or tumor necrosis factor-related apoptosis-inducing ligand (TRAIL), upon ligand binding active caspase-8 which was shown to cleave directly and then activate the caspase-3 protease effector, which in turn activates other substrates either directly or indirectly to finally stimulate apoptosis. On the other 
hand, the intrinsic or mitochondrial apoptotic pathway is associated with the activation of proteins such as Bcl-2 associated $\mathrm{X}$ (Bax) that belongs to the B-cell lymphoma-2 (Bcl2) family. These proteins cause mitochondrial disruption and then release of pro-apoptotic mitochondrial factors such as Cytochrome-c which interacts with apoptotic protease activating factor-1(Apaf-1) and activates caspase-9 (initiator caspase), which in turn proteolytically activates caspase-3 down-stream. Caspase- 8 activation through the binding of the death receptor can also trigger the mitochondrial pathway via $\mathrm{BH} 3$ interacting-domain death agonist (Bid), a pro-apoptotic member of the Bcl-2 family. Mitochondrial pathway activation is believed to amplify death-receptor- induced apoptosis process [19].

The porteolytic caspase-3 enzyme is considered a central effector caspase, which becomes active at relatively late stages and unites both the extrinsic and intrinsic pathways of apoptosis. The major importance of caspase-3 stimulated researchers to develop various sensors for its activity. In particular, many genetically encoded sensors that are used to monitor the activation of caspase- 3 in live cells at the single-cell level were developed [20,21].Accordingly, caspases play a crucial role in the activation and propagation of apoptotic signaling, and they are divided into three groups in terms of apoptosis and inflammation, namely initiator caspases, inflammatory caspases and effector caspases [22,23].The markers of apoptosis like active caspase-3, DNA fragmentation and others were documented to exist in seminal fluid from fertile men with normal semen parameters. However, the levels of these markers are noticeably increased in many occasions of spermatic disorders or disrupted spermatogenesis including subfertility and infertility. It has been found that in oligozoospermic patients, spermatogonia death by mitochondrial apoptotic damage additionally to meiosis malfunctioning, might be strongly associated to the decreased sperm output [24].Programmed cell death is a normal physiological phenomenon that maintains the number of germ cells within the supportive capacity of the Sertoli cells. Ejaculated spermatozoa display various apoptosis-like characteristics as in somatic cells; these apoptosis-related features do not necessarily indicate apoptosis death functions. Nevertheless, apoptosis-like phenotype in ejaculated sperm has been correlated with the presence of abnormal spermatozoa in seminal fluid. So that the failure to expel these abnormal spermatozoa during spermatogenesis process also termed as "abortive apoptosis" may be the reason for their presence in seminal fluid [25].

This study aimed to evaluate and assessment the apoptosis marker (caspase-3) and TAC among groups of Iraqi men with different versions of idiopathic infertility in comparison with a group of fertile men.

\section{Patients and Methods}

Ninety males were participated in the study including 70 males complaining of idiopathic infertility and 20 males with proven fertility 
as control. The infertile men were with age range of 18-50 years and were recruited to the IVF \& infertility center in Kamal AlSamarae hospital in Baghdad and Al-Sadder Medical City Hospital in Al-Najaf (Iraq) for the period from August 2016 to March 2017. These were divided into three groups; 25 oligozoospermia, 25 asthenozoospermia and 20 teratozoospermia. All participants were non-paid volunteers and a written informed consent was obtained from every subject. Exclusion criteria were; specific genital diseas $\neg$ es (infectious or non-infectious), undescended testes, systemic disease and testicular atro $\neg$ phy. All seminal fluid samples were collected by masturbation in sterile polypropylene containers after 3-5 days of abstinence of any sexual activity. Seminal fluid specimens were liquefied at conditions (temperature $37^{\circ} \mathrm{C}$ for 30 minutes) and then routine seminal fluid analysis was carried out accordᄀing to WHO criteria [26]. Serum samples were collected during a routine procedure of drawing venous blood without anticoagulant. Seminal plasma samples were collected from completely liquefied ejaculates and were centrifuged at $300 \mathrm{~g}$ for 20 minutes. A routine semen fluid analysis was performed upon liquefaction according to WHO Manual [27], to measure volume, appearance, $\mathrm{pH}$, fluidity and basic sperm parameters. The routine seminal fluid analysis was carried out estimating sperm density, motility, viability and morphology. The remaining seminal fluid sample was centrifuged at $1000 \times \mathrm{g}$ for $10 \mathrm{~min}$; the seminal plasma was separated for three equal parts and then stored at $-70{ }^{\circ} \mathrm{C}$ until further investigations. Solid phase immune-assays were used for quantitative measure of caspase-3(competitive ELISA kit from MyBiosource,USA) and TAC (sandwich ELISA kit from MyBiosource,USA) in seminal plasma.

\section{Statistical Analysis}

The program of Statistical Package for Social Sciences (SPSS) version 20 was used to measure mean \pm SD $95 \%$ confidence interval for mean values, and two extreme values, minimum and maximum. Odds ratio was applied for measuring strength of association. We set $\mathrm{P}$ value $\leq 0.05$ as significant value.

\section{Results}

Table (1) represent panoramic presentation of the study results in all study groups concerning semen quality parameters, TAC and Caspase- 3 levels in seminal plasma. The mean values $(\mathrm{U} / \mathrm{ml}) \pm \mathrm{SD}$ of the TAC were $28.47 \pm 9.66,23.46 \pm 6.62,14.08 \pm 4.21$ and $65.75 \pm 8.93$ in oligozoospermic, asthenozoospermic, teratozoospermic and control groups respectively. Results shows that the highest mean value was accounted in control group, and the lowest mean value was in teratozoospermia group. Positive correlation and highly significant differences were observed between sperm quality parameters and TAC levels in all study groups.

The results of caspase-3 level in seminal plasma revealed mean values $(\mathrm{ng} / \mathrm{ml}) \pm \mathrm{SD}$ of $2.99 \pm 2.05,2.13 \pm 1.10,3.06 \pm 2.19$ and $1.21 \pm 0.24$ in oligozoospermic, 
Role of Caspase -3 and Total Antioxidant Capacity (TAC) in Idiopathic Infertile Male

Madha Mohammed Sheet Saleh

asthenozoospermic, teratozoospermic and group, whereas the lowest was noticed in control groups respectively. The highest asthenozoospermia group of the idiopathic mean level was noticed in teratozoospermia infertile men.

Table (1): Semen parameters, TAC and Caspase-3 mean values in study groups in comparison with control group.

\begin{tabular}{|c|c|c|c|c|c|}
\hline \multirow{2}{*}{$\begin{array}{l}\text { Semen Parameters } \\
\text { and study markers }\end{array}$} & \multicolumn{3}{|c|}{ Men With Idiopathic infertility } & \multirow{2}{*}{$\begin{array}{l}\text { Fertile Men } \\
\qquad(n=20) \\
\text { Mean } \pm \text { SD }\end{array}$} & \multirow[b]{2}{*}{ P-value } \\
\hline & $\begin{array}{c}* \text { Olig }(n=25) \\
\text { Mean } \pm \text { SD }\end{array}$ & $\begin{array}{l}* * A s t h(n=25) \\
\text { Mean } \pm \text { SD }\end{array}$ & $\begin{array}{c}* * * \text { Terato }(\mathrm{n}=20) \\
\text { Mean } \pm \text { SD }\end{array}$ & & \\
\hline Volume (ml) & $2.03 \pm 0.71$ & $2.18 \pm 0.58$ & $2.85 \pm 0.93$ & $3.7 \pm 0.85$ & $\mathrm{HS}^{*}$ \\
\hline Sperm Count $\times 10^{6}$ & $5.32 \pm 2.08$ & $47.16 \pm 15.97$ & $41.55 \pm 12.28$ & $48.6 \pm 12.95$ & $\mathrm{HS}^{*}$ \\
\hline $\begin{array}{l}\text { Active linear prog- } \\
\text { ressive motility ( \% ) }\end{array}$ & $43.24 \pm 8.61$ & $10.60 \pm 4.16$ & $41.35 \pm 5.23$ & $41.55 \pm 5.59$ & $\mathrm{HS}^{*}$ \\
\hline $\begin{array}{l}\text { Sperm with normal } \\
\text { morphology ( } \%)\end{array}$ & $57.60 \pm 12.59$ & $59.00 \pm 12.25$ & $3.3 \pm 0.66$ & $65.75 \pm 8.93$ & $\mathrm{HS}^{*}$ \\
\hline $\mathrm{TAC}$ in ${ }^{* * * *} \mathrm{SP}(\mathrm{U} / \mathrm{ml})$ & $28.47 \pm 9.66$ & $23.46 \pm 6.62$ & $14.08 \pm 4.21$ & $60.36 \pm 10.73$ & "HS* \\
\hline $\begin{array}{c}\text { Caspase-3 level } \\
(\mathrm{ng} / \mathrm{ml}) \text { in seminal } \\
\text { plasma }\end{array}$ & $2.99 \pm 2.05$ & $2.13 \pm 1.10$ & $3.06 \pm 2.19$ & $1.21 \pm 0.24$ & HS * \\
\hline
\end{tabular}

*Olig= Oligozoospermia, ${ }^{* *}$ Asth=Asthenozoospermia, $* * *$ Terato=Teratozoospermia, ${ }^{* * * * S P}=$ Seminal plasma.

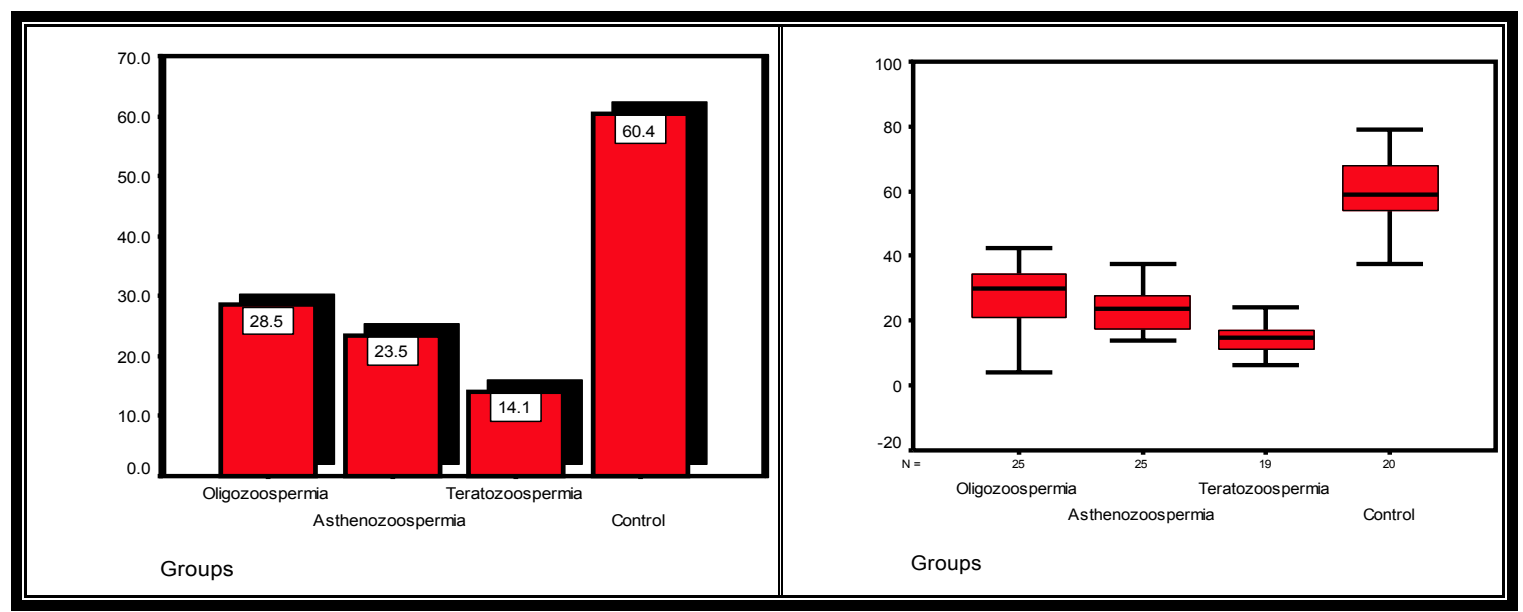

Figure (1): Mean values and Stem - Leaf plot of TAC (U/ml) in seminal plasma of idiopathic infertile male and control groups. 


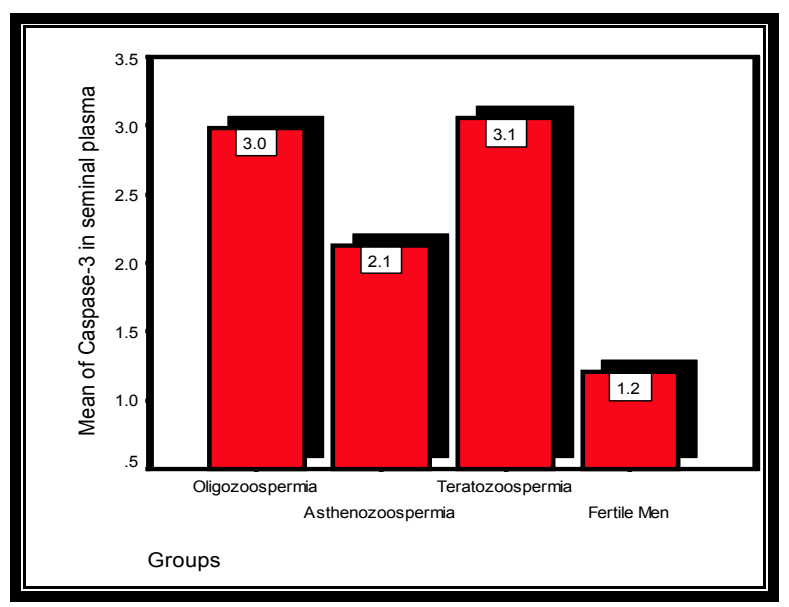

Figure (2): Mean values of caspase-3 (ng/ml) in seminal plasma of idiopathic infertile male and control groups.

Figures (1\&2) represent graphically and stem-leaf plot of the mean values for studied readings of TAC (U/ml) and caspase-3 $(\mathrm{ng} / \mathrm{ml})$ in seminal fluid of idiopathic infertile male and control groups.

\section{Discussion}

The positive correlation between the TAC and the semen quality parameters manifested in the current study was also noticed and in accordance with some previous studies [2830]. Such consistency in this issue let us to announce it as a fact and to consider it as the number one factor in the predisposition towards men idiopathic infertility among Iraq men with great similarities to other men in the world. Accordingly, TAC assay may be useful as specific marker for evaluation the oxidative stress status in seminal fluid.

The current study in agreement with study of Fazeli and Salimi who founded that seminal TAC levels were significantly lower in men with idiopathic infertility than in fertile men $(\mathrm{P}$ value $<0.01)$. A positive correlation was shown between semen parameters and TAC levels in men with idiopathic infertility [31].

The current study is highly consistent with that seminal oxidative stress could be one of the main factors in the pathogenesis of idiopathic infertility. Principally, high lev els of seminal ROS might lead to sperm DNA damage and lipid peroxidation, and eventually loss of sperm quality [32, 33]. Principally, understanding the sperm DNA fragmentation (SDF) causes considered is a goal sought over the last two decades, given the crucial importance of sperm DNA integrity for human reproduction as well as the health of the off-spring.

Muratori et.al concluded in results of their study various concept that included: (i) apoptosis process is the major pathway leading to DNA breakage in spermatozoa ;(ii) the immaturity of chromatin induces sperm DNA fragmentation (SDF) process through triggering of an apoptotic 
mechanism; and (iii) the attack of oxidative stress appears to act after spermiation, occurring mostly in live sperm. By conclusion, most live cells with active caspase enzyme also exhibited a high concentration of $8-\mathrm{OHdG}$ as a representative for oxidative stress, these suggesting activation of apoptotic pathways in oxidative-injured live cells[34].

Almeida et al. reported that spermatozoa with high activity of caspase- 3 seems to be linked with teratozoospermia and asthenozoospermia cases, thus suggesting that nuclear, mitochondrial and cytoskeletal abnormalities induce caspase-3 activation during spermiogenesis or sperm maturation process. Programmed cell death process is under the control of caspase and cysteine proteinase enzymes that cleave key cellular proteins after aspartate residues. Caspases enzymes are resident in cells as precursor proenzymes and become activated by several mechanisms. These mechanisms of apoptosis regulate normal spermatogenesis process. In the postnatal period, it is responsible for the death and phagocytosis of pre-meiotic germ cells in order to adjust their number to the number of Sertoli cells, and for the removal of damaged and abnormal germ cells during active spermatogenesis in the post-pubertal period [35].

A significant positive correlation between increased sperm damage by ROS and higher levels of the pro-apoptotic molecules such as caspases-3,9 and cytochrome $\mathrm{C}$ was also found in infertile individuals compared to healthy individuals indicating increased apoptosis, mitochondrial injury and finally DNA damage [36].These evidences revealed that mechanism of caspase-dependent apoptotic can originate in the within the mitochondria or in the cytoplasmic droplet and function in the nucleus Disruption in apoptotic balance due to oxidative stress considered a major cause of the weakness in sperm motility. Apoptotic process mediators such as caspase enzymes have been shown to occur at high levels in sperms with impaired motility. A positive significant correlation between caspase activity in the sperm midpiece and the DNA fragmentation has been shown in the low motility fractions of patients [37,38].

Recent evidences demonstrated that the most influential cause of damage in sperm DNA is oxidative stress. Such events are accompanied by impaired spermiogenesis process and increased ROS production. Disturbance of spermiogenesis process is probably caused by oxidative stress resulting impairment of protein translation and deficient germ cell. Also, protamination process when insufficient resulting poor compaction of DNA which renders it vulnerable to oxidative stress. Abnormal sperm cells may prematurely enter programmed cell death, which is accompanied by the activation of mitochondrial ROS generation. As a consequence of this cascade of events, in addition to the disruption of sperm production, the spermatozoa loss their motility and exhibit oxidative DNA damage that leads to DNA fragmentation [39]. 
Manente et al. demonstrated that a positive significant correlation was found between caspase- 3 activation and increased process of DNA fragmentation in patients with abnormal semen parameters [40].

\section{Conclusion}

The level of TAC in seminal fluid was lowered in idiopathic infertile male in comparison with control subjects indication an over accumulation of ROS in idiopathic infertile men. A positive relationship was revealed between sperm count, sperm moᄀtility as well as normal sperm morphology, with TAC levels in both idiopathic infertile men and fertile men. Therefore, TAC could be used as oxidative stress markers, and its assay is suggestively useful in the evaluation of idiopathic in $\neg$ fertility. In addition, these markers may guide us in anti-oxidant therapy which is still under field trials and the output about it is still controversial. Assessing these parameters would open a wide door on therapeutic trials for infertility associated with such parameter disorders. Also, the current study showed that seminal plasma levels of activated caspase -3 that considered as biomarkers of extrinsic and intrinsic DNA fragmentation were significantly increased among a group of Iraqi men with idiopathic infertility in comparison with its counterpart level among a group of men with normal fertility. This result guide us to think about how to exploit such correlation as a therapeutic tool by reducing the level of this activated caspase 3 through genetic manipulation (possible?) or initiation of intracellular anti-caspase-3 antibodies (intrabodies) in order to improve the semen parameters quality presumably that the low quality semen parameters are due to (partially or completely) the increased activated caspase-3 level.

\section{References}

[1] Alam N. Male factor infertility-basics revisited. J Coll Physicians Surg Pak ;2009, 19(4):205-6.

[2] Khatoon M, Chaaudhari AR, and Singh R . Effect of gender on anti-sperm antibodies in infertile couple in central India. Indian Journal of physiology and pharmacology;2012, 56(3): 262-266.

[3] Feng HL,Han YB, Sparks AET, and Sandlow JI. Characterization of Human Sperm Antigens Reacting with Anti-Sperm Antibodies from an Infertile Female Patient's Serum. J. Andrology;2008, 29(4):440-448. [4] Agarwal A, Virk G, Ong C, du Plessis SS. Effect of oxidative stress on male reproduction. World. J. Mens. Health;2014, 32(1):1-17.

[5] Aitken RJ, De Iuliis GN, Finnie JM, Hedges A, and McLachlan RI.Analy $\neg$ sis of the relationships between oxidative stresses, DNA damage and sperm vitality in a patient population: development of diᄀagnostic criteria. Hum Reprod;2010, 25(10): 24152426.

[6] de Lamirande E, Jiang H, Zini A, Kodama H, and Gagnon C . Reactive oxygen species and sperm physiology. Rev. Reprod;1997, 2(1):48- 54.

[7] Saleh RA, Agarwal A, Sharma RK, Nelson DR, and Thomas AJ. Effect of 
cigarette smoking on levels of seminal oxidative stress in infertile men: a prospective study. Fertil. Steril;2002, 78(3): 491-499.

[8] Sanocka D, and Kurpisz M . Reactive oxygen species and sperm cells. Reprod Biol Endocrinol;2004, 2(12):1-7.

[9] Paradisi R, Capelli M, Mandini M, Bellavia E, and Flamigni C . Increased levels of interferon-gamma in seminal plasma of infertile men. Andrologia; 1996,28,157-161. [10] Dousset B, Hussenet F, Daudin M, Bujan L, Foliguet B, and Nabet P. Seminal cytokine concentrations (IL-1b, IL-2, IL-6, sRIL-2, sRIL-6), semen parameters and blood hormonal status in male infertility. Human Reproduction;1997, 12(7): 14761479.

[11] Keskes-Ammar L, Feki-Chakroun N, Rebai T, Sahnoun, Z, Ghozzi H, Hammami S, Zghal K, Fki H, Damak J. and Bahloul A. Sperm oxidative stress and the effect of an oral vitamin $\mathrm{E}$ and selenium supplement on semen quality in infertile men. Arch. Andrology;2003, 49 (2): 83-94.

[12] Dawson EB, Harris WA, Teter MC, and Powell LC . Effect of ascorbic acid supplementation on the sperm quality of smokers. Fertil. Steril;1992, 58(5): 10341039.

[13] Greco E, Iacobelli M, Rienzi, L, Ubaldi F, Ferrero S, and Tesarik J . Re 7 duction of the incidence of sperm DNA fragmentation by oral antioxidant treatment. J. Androl.;2005, 26(3): 349-353.

[14] Wong WY, Merkus HM, Thomas CM, Menkveld R, Zielhuis GA, and Steegers-
Theunissen RP . Effects of folic acid and zinc sulfate on male factor subfertility: a double-blind, randomized, placebocontrolled trial. Fertil. Steril;2002, 77(3): 491-498.

[15] Hassan M, Watari H, Abu-Almaaty A, Ohba Y, and Sakuragi N. Apoptosis and Molecular Targeting Therapy in Cancer. BioMed Research International;2014, 150845: 23.

[16] Johnsen H L, and Horvitz H R. Both the apoptotic suicide pathway and phagocytosis are required for a programmed cell death in Caenorhabditis elegans. BMC Biology;2016, 14(39):1-14:1.

[17] Li F, Cao B, Ge JF, Zhao J, Jiang D, and Zheng SY . Expression of caspase-3 gene in gastric adenocarcinoma cell line SGC-7901 via Ad-FasL. Int. J. Clin. Exp. Pathol.;2015, 9(1):357-362.

[18] Riedl SJ, and Shi Y. Molecular mechanism of caspase regulation during apoptosis. Molecular Cell Biology;2014, 5: 897-907.

[19] Reyes-Zurita FJ, Rufino-Palomares EE, García-Salguero L, Peragón J, Medina PP, Parra A, Cascante $\mathrm{M}$, and Lupiáñez JA. Maslinic acid, a natural triterpene, induces a death receptor-mediated apoptotic mechanism in caco-2 p53-deficient colon adenocarcinoma cells. PLOS ONE;2016, 116 .

[20] Cardona M, López JA, Serafín A, Rongvaux A, Inserte J, García-Dorado D, Flavell R, Llovera M, Cañas X, Vázquez J, and Sanchis D . Executioner Caspase-3 and 7 Deficiency Reduces Myocyte Number in the 
Developing Mouse Heart. PLOS ONE;2015, 29:1- 21.

[21] Zlobovskaya OA, Sergeeva TF, Shirmanova MV, Dudenkova VV, Sharonov GV, Zagaynova EV, and Lukyanov KA . Genetically encoded far-red fluorescent sensors for caspase-3 activity, Reports; 2016, 60(2): 62-68:62.

[22] Dozic B, Glumac S, Boricic N, Dozic M, Anicic B, and Boricic I. Immunohistochemical expression of caspases 9 and 3 in adenoid cystic carcinoma of salivary glands and association with clinicopathological parameters. JBUON;2016, 21(1):152-160:153 .

[23] Lavrik IN, Golks A, and Krammer PH. . Caspases: pharmacological manipulation of cell death. J. Clin. Invest.;2005, 115: 26652672.

[24] Zhang HB, Lu SM, Ma CY, Wang L, Li $\mathrm{X}$, Chen ZJ . Early apoptotic changes in human spermatozoa and their relationships with conventional semen parameters and sperm DNA fragmentation. Asian J Androl., $2008,10(2): 227-35$.

[25] Sakkas D, Moffatt O, Manicardi GC, Mariethoz E, Tarozzi N, and Bizzaro D . Nature of DNA damage in ejaculated human spermatozoa and the possible involvement of apoptosis. Biol. Reprod.;2002, 66: 10611067.

[26] WHO (2010). Laboratory Manual for the examination of human semen and semen cervical mucus counteraction. WHO. Cambridge University Press, 3rd Edition. [27] Colagar AH, Karimi F, Jorsaraei SG . Correlation of sperm parameters with semen lipid peroxidation and total anti-oxidants levels in astheno- and oligoasthenoteratospermic men. Iran Red Crescent Med J;2013, 15(9): 780-785.

[28] Mahfouz R, Sharma R, Sharma D, Sabanegh E, and Agarwal A . Diag $\neg$ nostic value of the total antioxidant capacity (TAC) in human seminal plasma. Fertil. Steril.;2009, 91(3): 805-811.

[29] Pahune PP, Choudhari AR, and Muley PA.The total antioxidant power of semen and its correlation with the fertility potential of hu $\neg$ man male subjects. J. Clin. Diagn. Res;2013, 7(6): 991-995.

[30] Omran HM, Bakhiet M, Dashti MG. DNA integrity is a critical molecular indicator for the assessment of male infertility. Mol. Med. Rep.;2013, 7(5): 16311635.

[31] Fazeli,M and Salimi, S . Correlation of Seminal Total Antioxidant Capacity and Malondialdehyde Levels with Sperm parameters in Men with Idiopathic Infertility, Aviccena. J. Med. Biochem.;2016,4(1): 1-7. [32] Khosrowbeygi A, and Zarghami N. Levels of oxidative stress biomarkers in seminal plasma and their relationship with seminal parameters: Research Article. BMC Clinical Pathology;2007, 7(6):1-6.

[33] Pasqualotto FF, Sharma RK, Pasqualotto EB, and Agarwal A. Poor se $\neg$ men quality and ROS-TAC scores in patients with idiopathic infertility. Urol Int.;2008, 81(3): 263-270.

[34] Muratori M, Tamburrino L, Marchiani S, Cambi M, Olivito B, Azzari C, Forti G, and Baldi E. Investigation on the Origin of 
Sperm DNA Fragmentation: Role of Apoptosis, Immaturity and Oxidative Stress. Molecular Medicine;2015, 21:109-122, 116. [35] Almeida C, Cardoso MF, Sousa M, Viana P, Goncalves A, Silva J, and Barros A . Quantitative study of caspase-3 activity in semen and after swim-up preparation in relation to sperm quality. Human Reproduction;2005, 20(5):1307-1313, 1307. [36] Agarwal A, and Prabakaran SA . Oxidative stress and antioxidants in male infertility: a difficult balance: Review article. Iranian Journal of Reproductive Medicine;2005, 3(1): 1-8, 3.

[37] Weng SL, Taylor SL, Morshedi M, Schuffner A, Duran EH, Beebe S, and Oehninger S. Caspase activity and apoptotic markers in ejaculated human sperm. Mol. Hum. Reprod.;2002, 8: 984-991.
[38] Taylor SL, Weng SL, Fox P, Duran EH, Morshedi MS, Oehninger S, and Beebe SJ (. Somatic cell apoptosis markers and pathways in human ejaculated sperm: potential utility as indicators of sperm quality. Mol. Hum. Reprod.;2044,10: 825-834.

[39] Aitken RJ, and Curry BJ . Redox regulation of human sperm function: from the physiological control of sperm capacitation to the etiology of infertility and DNA damage in the germ line. Antioxid Redox Signal;2011, 14: 367-381. [40] Manente L, Pecoraro S, Picillo E, Gargiulo U, Gargiulo P, Deluca A, and Politano L . Molecular Evidence of Apoptotic Pathway Activation in Semen Samples with High DNA Fragmentation. In vivo;2015, 29: 289-294, 289. 